patients health education is performed in $35 \%$ of the center through periodic individual meetings.

Conclusion: Preliminary results confirm that the questionnaire is a valid tool to assess and compare patterns of care for pSS patients in terms of access and utilization of treatments and services across and within providers. Once available the questionnaires from all the centres, patientreported data linked with information from clinical records will allow to measure the patients journey more comprehensively along the care pathway and to identify best practices in terms of the level of perceived quality and the way the care is delivered and, moreover, opportunities for increasing value for patients.

Disclosure of Interests: : Chiara Seghieri: None declared, Chiara Baldini: None declared, Themis Exarchos: None declared, Luca Quartuccio: None declared, Elena Bartoloni Bocci: None declared, Roberta Priori: None declared, Francesco Carubbi: None declared, Francesco Ferro: None declared, Saviana Gandolfo: None declared, Stefano Bombardieri: None declared, Salvatore De Vita Grant/research support from: Roche, Pfizer, Abbvie, Novartis, BMS, MSD, Celgene, Janssen, Consultant for: Roche, Athanasios Tzioufas Grant/research support from: ABBVIE, PFIZER, AMGEN, NOVARTIS, GSK

DOI: $10.1136 /$ annrheumdis-2019-eular.6650

\section{SAT0208 TEN-YEAR OVERALL SURVIVAL AND STANDARIZED MORTALITY RATIO IN THE LARGEST SINGLE CENTER COHORT OF PATIENTS WITH PRIMARY SJOGREN'S ASSOCIATED LYMPHOMAS}

Marina Sikara, Dimitris Ziogas, Ourania Argyropoulou, Aristea Papageorgiou, Athanasios Tzioufas, Michael Voulgarelis. Athens School of Medicine, National and Kapodistrian University of Athens, Pathophysiology, Athens, Greece

Background: The development of non-Hodgkin's Lymphoma (NHL) in Sjögren's syndrome (SS) contributes to an inferior survival compared to SS patients without lymphoma.

Objectives: To record the long-term outcomes of SS patients with and without NHLs with 10-year survival curves and standardized mortality ratio (SMR) for first time in the literature.

Methods: In order to estimate the effect of NHL development in SS outcome, we expanded the follow-up time of the previously published cohort of SS-associated NHL cases and we retrospectively compared them with age- and sex-matched SS patients without NHL from our center. As outcome end-points, overall and event-free survivals (OS and EFS, respectively) were used. An event was defined as disease relapse or progression, histological transformation or death. Ten-year survival plots and SMR compared to General Greek population were calculated. The impact of rituximab in the outcome of SS associated NHL treated patients was also studied.

Results: From a total of 712 consecutive SS patients who fulfill the 2002 AECG classification criteria for Sjögren's, 77 were diagnosed with NHL. The prevalence of MALT and DLBC lymphoma in the total SS cohort was $7.1 \%$ and $1,7 \%$ respectively. The median follow-up time from SS diagnosis was 16.67 years (range: 4.00-38.91 years) corresponding to a total of 1283.71 person-years for SS-associated NHL patients and 17.92 years (range: $2.00-34.00$ years) with a total of 1380 person-years for SS patients without NHL. The median time from SS to lymphoma diagnosis was 5.5 years (range: $0-38$ years) and the median follow-up time after lymphoma diagnosis was 8.2 years (range: 0.1-26.2 years) months. During follow-up, 15 patients from the SS-NHL group died, 11 of whom due to lymphoma-related causes, while only 2 patients died in the control group (one due to advanced ovarian cancer and one due to cardiac arrest) $(\mathrm{p}=0.0013)$. Patients with $\mathrm{SS}$ associated $\mathrm{NHL}$ displayed a significantly higher risk of death $(\mathrm{HR}=5.95,95 \% \mathrm{Cl}: 1.66-21.38$, log-rank test, $\mathrm{p}=0.0006$ ) compared to their counterparts without lymphoma. The corresponding age-adjusted SMR of SS with and without NHLs versus the general Greek population was $7.94(95 \% \mathrm{Cl} 6.82-9.06)$ and $1.06(95 \% \mathrm{Cl}$ 0.06-2.18) respectively. In addition, 18 patients with SS-associated $\mathrm{NHL}$ $(23.38 \%)$ experienced lymphoma relapse or progression and $2(2.6 \%)$ lymphoma transformation; The vast majority of recorded events (27 of 29 events) were observed within the first ten years from NHL diagnosis and DLBCL was associated with the worst outcome; The 10-year EFS\% and OS\% for DLBC was $22.22 \%$ and $48.61 \%$ respectively while for MALTL were $68.15 \%$ and $82.03 \%$, respectively. Interestingly, the introduction of rituximab in the anti-lymphoma chemotherapeutic regimens didn't offer any long-term survival benefit.

Conclusion: Herein, we present the longest, to our knowledge, follow-up time of patients with SS-associated NHL; The majority of events that determine the morbidity and mortality of SS-NHL patients have already occurred within the first ten years from NHL diagnosis. In individuals with
SS, the evolution of NHL was found to have a detrimental effect in patients' survival. Among them, DLBCL patients experienced the worst outcome.

Disclosure of Interests: Marina Sikara: None declared, Dimitris Ziogas: None declared, Ourania Argyropoulou: None declared, Aristea Papageorgiou: None declared, Athanasios Tzioufas Grant/research support from: ABBVIE, PFIZER, AMGEN, NOVARTIS, GSK, Michael Voulgarelis: None declared

DOI:10.1136/annrheumdis-2019-eular.5794

\begin{tabular}{l|l}
\hline SAT0209 & A SIMPLIFIED APPROACH FOR SLE PATIENTS TO \\
REPORT DISEASE ACTIVITY USING A REVISED \\
VERSION OF THE SWEDISH SYSTEMIC LUPUS \\
ACTIVITY QUESTIONNAIRE
\end{tabular}

Susanne Pettersson ${ }^{1,2}$, Vera lllescas-Bäckelin ${ }^{3}$, Andreas Jonsen ${ }^{4}$, Iva Gunnarsson ${ }^{3}$, Estelle Trysberg ${ }^{5}$, Dag Leonard ${ }^{6}$, Christopher Sjowall ${ }^{7}$, Elisabet Svenungsson ${ }^{3} .{ }^{1}$ Karolinska Institute, Department of Neurobiology Care Sciences and Society, Stockholm, Sweden; ${ }^{2}$ Karolinska University Hospital, Inflammation and Infection Theme, Karolinska University Hospital, Stockholm, Sweden; ${ }^{3}$ Karolinska Institutet, Karolinska University Hospital, Rheumatology Unit, Department of Medicine, Solna, Stockholm, Sweden; ${ }^{4}$ Lund University, Department of Clinical Sciences Lund, Rheumatology, Lund, Sweden; ${ }^{5}$ Sahlgrenska University Hospital, Department of Rheumatology, Göteborg, Sweden; ${ }^{6}$ Academic Hospital, Rheumatology, Uppsala, Sweden; ' Linköping University, Rheumatology/Division of Neuro and Inflammation Sciences, Department of Clinical and Experimental Medicine, Linköping, Sweden

Background: The Systemic Lupus Activity Questionnaire (SLAQ) is a validated questionnaire, which captures patients' assessments of SLE-related symptoms and disease activity (1). However, it is extensive and in a recent study we found that some questions were difficult to answer, added little information, or had poor correlation with physicians' assessments (2). Thus, herein we revised the Swedish version of the questionnaire (SWE-SLAQr), building on previous results and we also asked patients for input. Our aim was to get an improved and shorter version, to support clinical work and online registries. The original SLAQ includes 26 items, while SWE-SLAQr includes 20 items.

Objectives: We compared patients' assessments of SLE disease activity, as reported in the SWE-SLAQr, with physicians' assessments using SLE activity measure (SLAM) and SLE disease activity index (SLEDAI-2K). In addition, we evaluated the performance of the symptom items of SWE SLAQr as compared to the corresponding items in SLAM.

Methods: Patients filled out SWE-SLAQr prior to physicians' assessments. Correlations between SWE-SLAQr-total, sub-scales (Symptom score, Patients global) and SLAM-excluding the 7 laboratory items (SLAM-nolab), SLAM and SLEDAI-2K as well as between the corresponding items in SLAQ and SLAM, were evaluated using Spearman's $\rho$.

Results: We included 101 patients, 85\% women, median age 43 (IQR 22) years, disease duration 14 (IQR 15) years. Patients reported more symptoms than recorded by doctors. Correlations between patients' and physicians' assessments were for SLAM-nolab: SWE-SLAQr total, $\rho=0.674$, Symptom score, $\rho=0.670$, and Patients global, $\rho=0.667$, as expected the correlations were lower for SLAM: SWE-SLAQr total, $\rho=0.472$, Symptom score, $\rho=0.467$, and Patients global, $\rho=0.501$. No correlations were found between patients' and physicians' assessments when using SLEDAI-2K $(\rho<0.09$ for all). Of symptom items fatigue $(\rho=0.741)$, alopecia $(\rho=0.695)$ and weight loss $(0.517)$ showed highest degree of correlation. Notably, symptoms of dyspnea/pleuritic chest pain had no correlation between patients' and physicians' assessments ( $\rho=0.152, \mathrm{p}=0.130)$. Conclusion: We conclude that SWE-SLAQr performed equally well as SLAQ (2), demonstrating that it can be used to monitor disease activity. We encourage further use of SWE-SLAQr and recommend its implementation in clinical care, we believe it is especially well suited to support digital and telephone contacts. However further attention is needed to evaluate the discrepancy between physicians' and patients' evaluation of thoracic pain/symptoms.

Table. Characteristics of participants

\begin{tabular}{lcc}
\hline & Median & IQR \\
\hline SWE-SLAQr total (0-37) & 8 & $3.5-13$ \\
Symptom score $(0-19)$ & 9 & $4-13.5$ \\
Patients global $(0-10)$ & 4 & $1-7$ \\
SLAM & 4 & $2-8$ \\
SLAM nolab & 3 & $1-6$ \\
SLEDAI-2K & 2 & $2-5.5$ \\
\hline
\end{tabular}




\section{REFERENCES}

[1] Karlson EW, et al. Validation of a Systemic Lupus Activity Questionnaire (SLAQ) for population studies. Lupus. 2003

[2] Pettersson S, et al. A comparison of patients' and physicians' assessments of disease activity using the Swedish version of the Systemic Lupus Activity Questionnaire. Scand J Rheumatol. 2017.

Disclosure of Interests: None declared

DOI: 10.1136/annrheumdis-2019-eular.3099

\section{SAT0210 THE ANTI-RO52 PREVALENCE IN THE SJÖGREN'S SYNDROME PICTURE: A SINGLE CENTER CROSS SECTIONAL STUDY}

Larissa Valor, Hannah Schenker, Melanie Hagen, Johannes Knitza, Jürgen Rech, Georg Schett. Friedrich-Alexander-University Erlangen-Nürnberg, Department of Internal Medicine 3 - Rheumatology and Immunology, Erlangen, Germany

Background: Sjögren syndrome (SjS) is an autoimmune disorder characterized by inflammation and destruction of exocrine glands. The presence of autoantibodies (AA) against the Ro52/TRIM21, an RNP complex binding to the stem-loop structure of human cytoplasmic RNA, might be relevant in the SjS pathogeny. It has been suggested that distinguishing between antibody reactivity against Ro60 and Ro52/TRIM21 could be helpful in terms of evaluating clinical course, features and even pre-symptomatic stages of the disease (1).

Objectives: To evaluate the prevalence of anti-Ro52/TRIM21 antibodies in a cohort of patients diagnosed with primary SjS.

Methods: In this cross-sectional study we evaluated 179 patients with primary SjS according to the ACR classification criteria who had been admitted between December 2008 and December 2018 to our outpatient clinic. All patients had ANA titers higher than 1:320 (2) in at least two positive determinations for any pattern. ANA, anti-Ro52/TRIM21, anti-Ro60, anti-La and rheumatoid factor (RF) were tested by immunoblot (Euroimmun, Lübeck, Germany).

Results: In our cohort the median age at diagnosis was 57 years (range: 20- 85 years) with a clear dominance of females $(n=160,89 \%)$. the most frequently reported ANA patterns were speckled $(93 \%)$, while only few patients had a homogeneous $(6 \%)$ pattern. $177 / 179$ were positive for anti-Ro52/TRIM21 (98\%), 159/179 (88\%) for anti-Ro60, 127/179 for antiLa $(79 \%)$ and $94 / 179(52 \%)$ showed RF reactivity. 76/179 (42\%) patients showed all four reactivities (anti-Ro52/TRIM21, anti-Ro60, anti-La and RF). Out of these 76 patients, $11(6 \%)$ patients exhibited Raynaud's syndrome, 25 (13\%) exhibited arthritis/arthralgia, 31 (17\%) had hypergammaglobulinemia, $13(7 \%)$ had hypocomplementemia and $26 \%$ had elevated free kappa/lambda chains, as typical clinical and laboratory features described in SjS.

Conclusion: Our results showed that anti-Ro52/TRIM21 but not anti-Ro60 is present in virtually all patients with SjS and had the most prevalent antibody reactivity. This finding needs to be considered in the current classification criteria of SjS (2), which include the presence of anti-Ro60, rather than anti-Ro52/TRIM21. Also, including the anti-Ro52/TRIM21 measurement in larger cohorts and longitudinal studies would also help us in improving the knowledge of its pathogenic role and to define of more focused diagnostic/therapeutic strategies.

\section{REFERENCES}

[1] Menendez A, Gomez J, Escanlar E, Caminal-Montero L, Mozo L. Clinical associations of anti-SSA/Ro60 and anti-Ro52/TRIM21 antibodies: Diagnostic utility of their separate detection. Autoimmunity. 2013; 46(1):32-9.

[2] Shiboski SC, Shiboski CH, Criswell L, et al. American College of Rheumatology classification criteria for Sjögren's syndrome: a data-driven, expert consensus approach in the Sjögren's International Collaborative Clinical Alliance cohort. Arthritis Care Res (Hoboken) 2012;64(4):475-87.

Disclosure of Interests: Larissa Valor: None declared, Hannah Schenker None declared, Melanie Hagen: None declared, Johannes Knitza: None declared, Jürgen Rech Grant/research support from: Bristol-Myers Squibb and Celgene (greater than $\$ 10,000$ ), Consultant for: Bristol-Myers Squibb, Celgene, Chugai, GlaxoSmithKline, Janssen, Eli Lilly, Novartis, Roche, Sanofi Aventis, and UCB (in total more than $\$ 10,000$ ), Speakers bureau: Bristol-Myers Squibb, Celgene, Chugai, GlaxoSmithKline, Janssen, Eli Lilly, Novartis, Roche, Sanofi Aventis, and UCB (in total more than \$10,000), Georg Schett: None declared

DOI: 10.1136/annrheumdis-2019-eular.3660

\section{SAT0211 LYMPHOPENIA WITH SYSTEMIC LUPUS ERYTHEMATOSUS: LYMPHOCYTE SUBSETS OF PATHOPHYSIOLOGICAL FEATURES}

Ning Yan ${ }^{1}$, Jie Liang ${ }^{1}$, Fangyuan $\mathrm{Hu}^{1}$, Chong Gao ${ }^{2}$, LI Xiaofeng ${ }^{1}$, Caihong Wang ${ }^{1}$. ${ }^{1}$ The Second Hospital of Shanxi Medical University, Taiyuan, China; ${ }^{2}$ Brigham and Women's Hospital, Boston, United States of America

Background: Systemic lupus erythematosus (SLE) is an autoimmune disease involving multiple systems. It is mainly characterized by abnormal activation of $T$ and $B$ lymphocytes and the production of a large number of autoantibodies. Blood system involvement is a common clinical manifestation. The occurrence of lymphopenia is very common, easy to merge infection and primary immunodeficiency ${ }^{[1-3]}$.

Objectives: To study the distribution characteristics of peripheral blood lymphocyte cell subsets in patients with newly diagnosed SLE and to explore the possible mechanism of lymphocyte reduction.

Methods: Sixty-six female patients with initial SLE. The diagnosis was consistent with the 1997 revised American College of Rheumatology (ACR) SLE classification criteria, and no glucocorticoids and immunosuppressive agents were used. According to the absolute value lymphocytes with SLE, 40 patients were divided into lymphopenia group(LA)(LY< $\left.1.0 \times 10^{9} / \mathrm{L}\right)$, and 26 patients were normal lymphocytes group(LN). 30 female healthy controls. The absolute numbers of lymphocyte cell subsets and $\mathrm{T}$ subsets (total $\mathrm{T}, \mathrm{CD} 4^{+} \mathrm{T}, \mathrm{CD} 8^{+} \mathrm{T}, \mathrm{T}$ helper 1 (Th1), $\mathrm{T}$ helper 2 (Th2), $\mathrm{T}$ helper 17 (Th17) and $\mathrm{CD}^{+} \mathrm{CD} 25^{+} \mathrm{Foxp}^{+} \mathrm{T}$ regulatory (Treg) cells) in peripheral blood were measured by Flow Cytometer (FCM).

Results: In the lymphopenia group, the absolute counts of $\mathrm{T}$ cell subsets and NK cells were significantly reduced, but the percentage of B cells and Th1 cells and the ratio of Th1/Th2 cells were significantly increased (Figure 1). The absolute number of CD4+ T cells and CD8+ T cells was significantly positively correlated with $\mathrm{C} 3$ levels(Figure 2).

Conclusion: Decresed T cell subsets and NK cells may be key pathophysiological features, as risk factor for development of severe infections and primary immunodeficiency in patients lymphopenia with SLE. Meanwhile, decreased CD4+ T cells and CD8+ T cells and increased B cells may cause auto- antibodies production leading to lymphopenia with SLE. Further studies are needed to assess profound lymphopenia and require specific management.
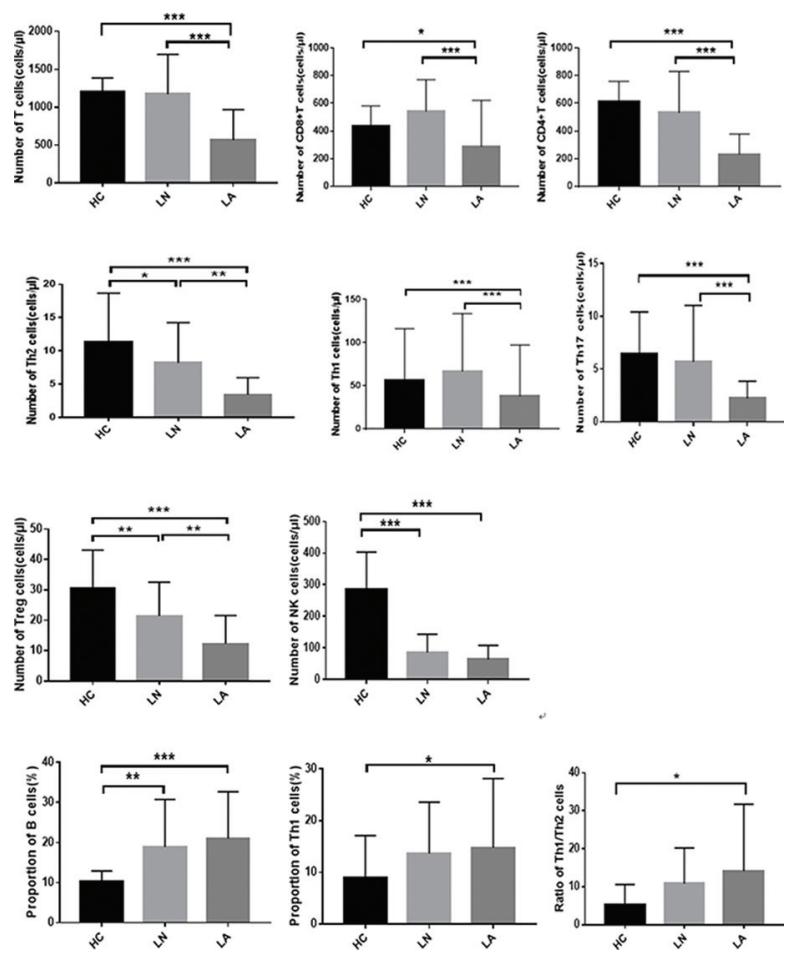

Figure 1. Absolute number and percentage of peripheral T cells, T-cells subsets, $B$ cells and NK cells of patients with HC, LN and LA. One-Way ANOA analysis was used to compare $\mathrm{HC}$ and $\mathrm{LN}$ and LA with $\mathrm{SLE}\left({ }^{*} \mathrm{p}<0.5,{ }^{* *} \mathrm{p}<0.01,{ }^{* *} \mathrm{p}<0.001\right)$. HC, healthy control; LN, lymphocyte normal; LA, lymphopenia. 